function mutations in mice and humans results in abnormal DYSF internalization $(10,16)$. Whether mislocalization of CAV3 and DYSF within the myofiber contributes to CNM disease progression remains to be determined. These data suggest that a common pathway links MTM1, DNM2, BIN1, DYSF, and CAV3 in the biogenesis and maintenance of muscle, specifically at the T-tubule. If DNM2 levels are found to be upregulated in these other forms of myopathy, then targeting DNM2 becomes a common therapeutic strategy for a wider range of muscle disease.

\section{Acknowledgments}

This work was supported by NIH grants NS047726, AR053646, and NS072027.

Address correspondence to: Elizabeth M. McNally, University of Chicago, 5841 S. Maryland, MC6088, Chicago, Illinois 60637, USA. Phone: 773.702.2684; Fax: 773.702.2681; E-mail: emcnally@ uchicago.edu.

1. Dowling JJ, Gibbs EM, Feldman EL. Membrane traffic and muscle: lessons from human disease.
Traffic. 2008;9(7):1035-1043.

2. De Matteis MA, Godi A. PI-loting membrane traffic. Nat Cell Biol. 2004;6(6):487-492.

3. Buj-Bello A, et al. The lipid phosphatase myotubularin is essential for skeletal muscle maintenance but not for myogenesis in mice. Proc Natl Acad Sci U S A. 2002;99(23):15060-15065.

4. Dowling JJ, et al. Loss of myotubularin function results in T-tubule disorganization in zebrafish and human myotubular myopathy. PLoS Genet. 2009;5(2):e1000372.

5. Macia E, Ehrlich M, Massol R, Boucrot E, Brunner C, Kirchhausen T. Dynasore, a cell-permeable inhibitor of dynamin. Dev Cell. 2006;10(6):839-850.

6. Ferguson SM, et al. Coordinated actions of actin and BAR proteins upstream of dynamin at endocytic clathrin-coated pits. Dev Cell. 2009;17(6):811-822.

7. Bitoun $\mathrm{M}$, et al. Mutations in dynamin 2 cause dominant centronuclear myopathy. Nat Genet. 2005;37(11):1207-1209.

8. Cowling BS, et al. Increased expression of wild-type or a centronuclear myopathy mutant of dynamin 2 in skeletal muscle of adult mice leads to structural defects and muscle weakness. Am J Pathol. 2011; 178(5):2224-2235.

9. Cowling BS, et al. Reducing dynamin 2 expression rescues $\mathrm{X}$-linked centronuclear myopathy. J Clin Invest. 2014;124(3):1350-1363.

10. Durieux AC, et al. A centronuclear myopathydynamin 2 mutation impairs skeletal muscle structure and function in mice. Hum Mol Genet. 2010; 19(24):4820-4836.

11. Kojima C, et al. Regulation of Bin $1 \mathrm{SH} 3$ domain binding by phosphoinositides. EMBO J. 2004; 23(22):4413-4422.

12. Royer B, Hnia K, Gavriilidis C, Tronchere H, Tosch $\mathrm{V}$, Laporte J. The myotubularin-amphiphysin 2 complex in membrane tubulation and centronuclear myopathies. EMBO Rep. 2013;14(10):907-915.

13. Buj-Bello A, et al. AAV-mediated intramuscular delivery of myotubularin corrects the myotubular myopathy phenotype in targeted murine muscle and suggests a function in plasma membrane homeostasis. Hum Mol Genet. 2008;17(14):2132-2143.

14. Lawlor MW, et al. Enzyme replacement therapy rescues weakness and improves muscle pathology in mice with X-linked myotubular myopathy. Hum Mol Genet. 2013;22(8):1525-1538

15. Takahashi K, Miyoshi H, Otomo M, Osada K, Yamaguchi N, Nakashima H. Suppression of dynamin GTPase activity by sertraline leads to inhibition of dynamin-dependent endocytosis. Biochem Biophys Res Commun. 2010;391(1):382-387.

16. Liu N, et al. Mice lacking microRNA 133a develop dynamin 2-dependent centronuclear myopathy. J Clin Invest. 2011;121(8):3258-3268.

17. Galbiati F, et al. Caveolin-3 null mice show a loss of caveolae, changes in the microdomain distribution of the dystrophin-glycoprotein complex, and t-tubule abnormalities. J Biol Chem. 2001; 276(24):21425-21433

18. Klinge L, et al. Dysferlin associates with the developing T-tubule system in rodent and human skeletal muscle. Muscle Nerve. 2010;41(2):166-173.

19. Demonbreun AR, Fahrenbach JP, Deveaux K, Earley JU, Pytel P, McNally EM. Impaired muscle growth and response to insulin-like growth factor 1 in dysferlin-mediated muscular dystrophy. Hum Mol Genet. 2011;20(4):779-789.

20. Lee E, et al. Amphiphysin 2 (Bin1) and T-tubule biogenesis in muscle. Science. 2002;297(5584):1193-1196.

21. Kerr JP, et al. Dysferlin stabilizes stress-induced $\mathrm{Ca} 2+$ signaling in the transverse tubule membrane. Proc Natl Acad Sci U S A. 2013;110(51):20831-20836.

\title{
PPAR $y$ in emphysema: blunts the damage and triggers repair?
}

\author{
Neil J. Kelly and Steven D. Shapiro
}

Division of Pulmonary, Allergy, and Critical Care, Department of Medicine, UPMC and University of Pittsburgh, Pittsburgh, Pennsylvania, USA.

Cigarette smoke is the most common cause of pulmonary emphysema, which results in an irreversible loss of lung structure and function. Th1 and Th17 immune responses have been implicated in emphysema pathogenesis; however, the drivers of emphysema-associated immune dysfunction are not fully understood. In this issue of the JCI, Shan and colleagues found that peroxisome proliferator-activated receptor $\gamma(\operatorname{PPAR} \gamma)$ is downregulated in APCs isolated from the lungs of emphysematous chronic smokers and mice exposed to cigarette smoke. Furthermore, treatment with a PPAR $\gamma$ agonist prevented emphysema development and appeared to reduce emphysemaassociated lung volume expansion in mice exposed to cigarette smoke. Further work will need to be done to evaluate the potential of PPAR $\gamma$ agonists to restore lung capacity in emphysematous patients.

Pulmonary emphysema is a major component of chronic obstructive pulmonary dis-

Conflict of interest: The authors have declared that no conflict of interest exists.

Citation for this article: J Clin Invest. 2014; 124(3):978-980. doi:10.1172/JCI74417. ease (COPD) and involves the loss of alveolar units distal to the terminal bronchioles. Even though COPD holds an unenviable position as the world's fourth-leading cause of death, current medical interventions have little to offer beyond symptomatic relief. Meanwhile, the prevalence of
COPD is expected to continue to rise as low- and middle-income countries join in the developed world's tobacco addiction. If we are to avoid this grim projection, we must expand our knowledge of the basic mechanisms behind lung injury and repair before translating these findings into novel therapeutic treatments.

\section{Pulmonary emphysema results from an imbalance between elastases and anti-elastases}

It has been more than 50 years since Laurell and Eriksson first identified a deficiency in $\alpha-1$ antitrypsin, the major inhibitor of neutrophil elastase (ELANE), as the culprit behind hereditary pulmonary emphysema (1). Since the involvement of ELANE in pulmonary emphysema was first reported, our understanding of the disease's 


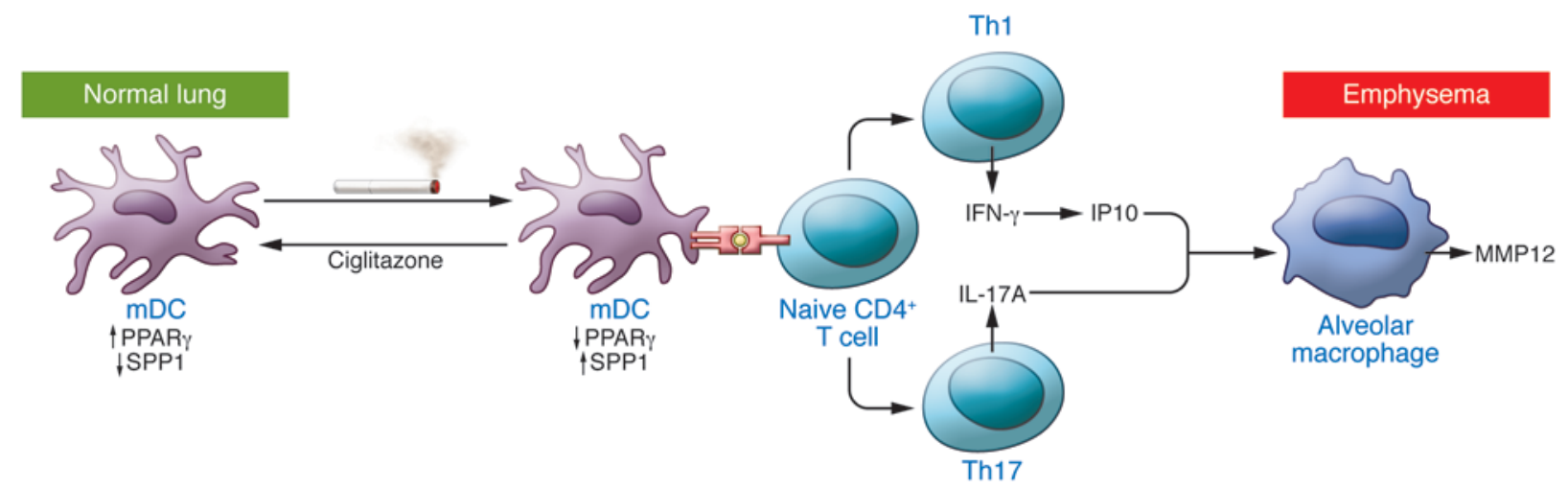

\section{Figure 1}

Proposed role of PPAR $\gamma$ in pulmonary emphysema pathogenesis. Smoking leads to the acquisition of a pathogenic phenotype in lung mDCs, characterized by ciglitazone-reversible downregulation of PPAR $\gamma$ and upregulation of SPP1. Pathogenic mDCs then direct the differentiation of Th1 and Th17 helper T lymphocytes, whose cytokines IFN- $\gamma$ and IL-17A, respectively, trigger the release of the macrophage elastase MMP12 and accompanying alveolar destruction.

pathogenesis has grown around a core paradigm, whereby a protease-antiprotease imbalance results in degradation of the elastic fibers that impart structural stability and compliance on the lung. Beginning with a report by Gross and colleagues (2), subsequent studies revealed that intratracheal instillation of elastases, including ELANE, reproduces the morphologic characteristics of emphysema in rodents. In addition to neutrophils and their associated elastases, the scope of emphysema research has expanded to include macrophages - the predominant inflammatory cells of the lung - and their elastolytic matrix metalloproteinases (MMPs). Mice lacking macrophage elastase (MMP12) exhibit total resistance to smoke-induced emphysema (3), and MMP12 appears to act synergistically with ELANE in vivo, with each elastase degrading the other's inhibitor. Ultimately, the cooperative actions of ELANE and MMP12 bring about the defining elastolysis and airspace enlargement of emphysema.

The implication that neutrophil- and macrophage-derived elastases influence emphysema pathogenesis has justifiably led to an intense research focus on innate immunity; however, the observed increase in $\mathrm{T}$ lymphocytes in the lungs of emphysema patients suggests that the adaptive immune system facilitates the innate response (4). Our understanding of the crosstalk between innate and adaptive immunity in emphysema is far from clear, partly because murine research has thus far shown an inconsistent role for lymphocytes in disease progression. For example, mice with deficient $\mathrm{T}$ and $\mathrm{B}$ cell responses devel- op full-blown emphysema in response to cigarette smoke (5), yet CD8-deficient mice are completely resistant to the same insult (6). However, IFN- $\gamma$ (through the action of the cytokine IP10) and IL-17A, the signature cytokines produced by $\mathrm{T}$ helper subsets Th1 and Th17, respectively, have the potential to link the adaptive and innate responses by mediating the release of MMP12 from alveolar macrophages $(7,8)$. In addition to being elevated in smoke-exposed lungs, IFN- $\gamma$ and IL-17A are strongly associated with autoimmune disorders (9), and their preponderance supports the idea that autoreactivity perpetuates the sustained decline of lung function observed in a subset of former smokers (10). In fact, some studies have shown that elastin fragments elicit a recall response in the peripheral $\mathrm{T}$ lymphocytes of emphysema patients (8), underscoring a potential link between autoimmunity and human emphysema.

\section{A link between smoking and autoimmunity}

But how does smoking lead to autoimmunity? In a series of reports, Shan and colleagues turned to APCs to address this question. In an initial human study (8), Shan et al. found that APCs isolated from emphysematous lungs induce naive $\mathrm{CD}^{+}$ $\mathrm{T}$ cells to differentiate into Th 1 and Th17 cells. In mouse models, which do not recapitulate the anti-elastin autoimmunity observed in human patients, Shan and colleagues showed that tobacco smoke exposure causes a phenotypic switch in lung APCs that is characterized by increased expression of the secreted phosphoprotein osteopontin (SPP1). These patho- genic APCs then direct the differentiation of Th1 and Th17 lymphocytes, mimicking the actions of APCs from human smokers (11). Furthermore, mice lacking IL-17A or SPP1 failed to develop airspace enlargement in response to long-term smoke exposure, highlighting the importance of APC-directed $\mathrm{T}$ cell differentiation in smoke-induced immune dysfunction.

Increasing evidence indicates that the nuclear hormone receptor PPAR $\gamma$, best known as a regulator of tissue metabolism and adipogenesis, plays a role in lung development and inflammation. In this issue of the JCI, Shan and colleagues (12) provide further support that PPAR $\gamma$ regulates immune responses in the lung, with an initial observation that PPAR $\gamma$ is downregulated in APCs isolated from human smokers with emphysema and from mice exposed to tobacco smoke. Additionally, deletion of Pparg in CD11 $\mathrm{c}^{+}$APCs, which include macrophages and myeloid dendritic cells (mDCs), caused spontaneous SPP1dependent airspace enlargement in mice. Coupled with their previous work, the current study by Shan and colleagues suggests that by dampening PPAR $\gamma$, tobacco smoke unleashes SPP1 to induce differentiation of Th1 and Th17 lymphocytes. When activated, Th1 and Th17 lymphocytes secrete IFN- $\gamma$ and IL-17A, respectively, triggering the synthesis and release of destructive elastases from alveolar macrophages (Figure 1).

\section{PPARY activation: a treatment for emphysema?}

Naturally, if functional antagonism of PPAR $\gamma$ by tobacco smoke worsens emphysema, then pharmacologic PPAR $\gamma$ activa- 
tion with the thiazolidinediones (TZDs) should abrogate disease progression. As expected, Shan and colleagues (12) demonstrated that the PPAR $\gamma$ agonist ciglitazone prevented murine emphysema despite active smoke exposure. Less definitively, mice exposed to cigarette smoke for 3 months followed by ciglitazone treatment exhibited an apparent restoration of lung volume, suggesting that TZDs are able to reverse the course of emphysema. Should these results be substantiated, TZDs would join a growing list of agents with the potential to reverse rodent emphysema. In the first report of emphysema reversal, Massaro and Massaro (13) demonstrated the initiation of new alveolar growth by retinoic acid (RA) following elastase-induced emphysema in rats. Using a more pertinent smoke exposure model, albeit with a less rigorous physiological assessment, Seimetz and colleagues (14) reported that inhibition of inducible nitric oxide synthase (NOS2) reverses emphysema in mice. In an interesting twist, $\mathrm{NO}$ has also been reported to increase the expression of SPP1 (15), suggesting that TZDs and NOS2 inhibitors may have an overlapping therapeutic mechanism.

In light of the findings by Shan and colleagues, as well as the results of a recent study (16) that describes airway inflammation and emphysema onset following expression of a dominant-negative PPAR $\gamma$ in alveolar epithelium, PPAR $\gamma$ appears to play a broad role in checking destructive inflammatory processes in the lung. Is it possible, then, that suppression of inflammation is enough to initiate murine lung regeneration? Perhaps, but it may be that TZDs themselves trigger lung regeneration. In any discussion of tissue regeneration, it is helpful to look to development for insight. Based on studies of children born to mothers who smoked during pregnancy, we know that smoking impairs alveogenesis (17), a process that begins at the end of gestation in humans and occurs entirely postnatally in rodents. While our knowledge is far from complete, it is already clear that PPAR $\gamma$ is a major regulator of this process. In the nascent lung, the loss of alveolar epithelial Pparg impairs development with consequent enlargement of the airspaces (18). Further, in utero nicotine exposure decreases the number of lipid-laden pulmonary lipofibroblasts, which are thought to be important for lung development, by promoting their transdifferentiation into myofibroblasts. However, these transdifferentiated myofibroblasts revert to lipofibroblasts in vitro upon treatment with TZDs (19), while in vivo TZD administration accelerates alveogenesis in rats during the peak period of lung development (20).

It is interesting to speculate that treatment of emphysematous adults with TZDs could initiate a similar program of elastic fiber assembly, which historically has been considered to be a limiting factor in repair of emphysema-associated lung damage. However, repair in rodent models does not necessarily translate to humans. For example, RA treatment, which is efficacious in rats, thus far has shown no potential to reverse emphysema in human trials (21). The greatest concern for repairing damaged lung tissue has less to do with the regenerative capability of stem cells and more to do with the restoration of the extracellular matrix and its elastic fiber cable network. Recapitulating the spatial and temporal regulation of the multiple steps required to regenerate the extracellular matrix in the adult lung is a daunting task; however, a recent case report (22) provides a glimmer of hope that alveogenesis may be possible in the fully developed human lung. As we move forward in the search for a medical cure for pulmonary emphysema, understanding and exploiting the mechanisms behind this phenomenon offers our best chance of success.

\section{Acknowledgments}

Neil J. Kelly is supported by NIH/National Heart, Lung, and Blood Institute (NHLBI) grant T32HL094295-04, and Steven D. Shapiro is supported by NIH/NHLBI grant 5P01HL103455-03.

Address correspondence to: Steven D. Shapiro, Office of the CMSO, UPMC, U.S. Steel Tower, 600 Grant Street, Suite 6250, Pittsburgh, Pennsylvania 15219, USA. Phone: 412.605.3995; Fax: 412.647.4801; E-mail: shapirosd@upmc.edu.

1. Laurell CB, Eriksson S. The electrophoretic $\alpha 1$-globulin pattern of serum in $\alpha 1$-antitrypsin deficiency. 1963. COPD. 2013;10(suppl 1):3-8.

2. Gross P, Pfitzer EA, Tolker E, Babyak MA, Kaschak M. Experimental emphysema: its production with papain in normal and silicotic rats. Arch Environ Health. 1965;11:50-58.
3. Hautamaki RD, Kobayashi DK, Senior RM, Shapiro SD. Requirement for macrophage elastase for cigarette smoke-induced emphysema in mice. Science. 1997;277(5334):2002-2004.

4. Saetta M, et al. CD8+ve cells in the lungs of smokers with chronic obstructive pulmonary disease. Am J Respir Crit Care Med. 1999;160(2):711-717.

5. D'hulst AI, et al. Cigarette smoke-induced pulmonary emphysema in scid-mice. Is the acquired immune system required? Respir Res. 2005;6:147.

6. Maeno T, Houghton AM, Quintero PA, Grumelli S, Owen CA, Shapiro SD. CD8 ${ }^{+} \mathrm{T}$ Cells are required for inflammation and destruction in cigarette smoke-induced emphysema in mice. J Immunol. 2007;178(12):8090-8096.

7. Grumelli S, et al. An immune basis for lung parenchymal destruction in chronic obstructive pulmonary disease and emphysema. PLoS Med. 2004; 1(1):e8.

8. Shan $M$, et al. Lung myeloid dendritic cells coordinately induce $\mathrm{TH} 1$ and $\mathrm{TH} 17$ responses in human emphysema. Sci Transl Med. 2009; 1(4):4ra10

9. Palmer MT, Weaver CT. Autoimmunity: increasing suspects in the $\mathrm{CD}^{+} \mathrm{T}$ cell lineup. Nat Immunol. 2010;11(1):36-40.

10. Anthonisen NR, Connett JE, Murray RP. Smoking and lung function of Lung Health Study participants after 11 years. Am J Respir Crit Care Med. 2002;166(5):675-679.

11. Shan $\mathrm{M}$, et al. Cigarette smoke induction of osteopontin (SPP1) mediates $\mathrm{T}(\mathrm{H}) 17$ inflammation in human and experimental emphysema. Sci Transl Med. 2012;4(117):117ra119.

12. Shan M, et al. Agonistic induction of PPAR $\gamma$ reverses cigarette smoke-induced emphysema. J Clin Invest. 2014;124(3):1371-1381.

13. Massaro GD, Massaro D. Retinoic acid treatment abrogates elastase-induced pulmonary emphysema in rats. Nat Med. 1997;3(6):675-677.

14. Seimetz $M$, et al. Inducible NOS inhibition reverses tobacco-smoke-induced emphysema and pulmonary hypertension in mice. Cell. 2011; 147(2):293-305.

15. Guo H, Cai CQ, Schroeder RA, Kuo PC. Osteopontin is a negative feedback regulator of nitric oxide synthesis in murine macrophages. J Immunol. 2001; 166(2):1079-1086.

16. Wu L, Wang G, Qu P, Yan C, Du H. Overexpression of dominant negative peroxisome proliferator-activated receptor-gamma (PPAR $\gamma$ ) in alveolar type II epithelial cells causes inflammation and T-cell suppression in the lung. Am J Pathol. 2011;178(5):2191-2204.

17. Maritz GS, Dennis H. Maternal nicotine exposure during gestation and lactation interferes with alveolar development in the neonatal lung. Reprod Fertil Dev. 1998;10(3):255-261.

18. Simon DM, et al. Epithelial cell PPAR $\gamma$ contributes to normal lung maturation. FASEB J. 2006; 20(9):1507-1509.

19. Rehan VK, Sakurai R, Wang Y, Santos J, Huynh K, Torday JS. Reversal of nicotine-induced alveolar lipofibroblast-to-myofibroblast transdifferentiation by stimulants of parathyroid hormone-related protein signaling. Lung. 2007;185(3):151-159.

20. Wang Y, et al. Peroxisome proliferator-activated receptor gamma agonists enhance lung maturation in a neonatal rat model. Pediatr Res. 2009; 65(2):150-155

21. Roth MD, et al. Feasibility of retinoids for the treatment of emphysema study. Chest. 2006; 130(5):1334-1345.

22. Butler JP, Loring SH, Patz S, Tsuda A, Yablonskiy DA, Mentzer SJ. Evidence for adult lung growth in humans. NEngl J Med. 2012;367(3):244-247. 